\title{
Oriented Arrays of Iron Nanowires: Synthesis, Structural and Magnetic Aspects
}

Anna S. Goncharova ${ }^{a,}{ }^{*}$, Stepan V. Sotnichuk ${ }^{\mathrm{a}}$, Anna S. Semisalova ${ }^{\mathrm{b}}$, Tatiana Yu. Kiseleva ${ }^{\mathrm{b}}$, Ilya Sergueev $^{c}$, Marcus Herlitschke ${ }^{c}$, Kirill S. Napolskii ${ }^{\mathrm{a}, \mathrm{d}}$, Andrei A. Eliseev ${ }^{\mathrm{a}, \mathrm{d}}$ ${ }^{\mathrm{a}}$ Department of Materials Science, M.V. Lomonosov Moscow State University, 119991, Moscow, Russia

${ }^{b}$ Department of Physics, M.V. Lomonosov Moscow State University, 119991, Moscow, Russia

${ }^{\mathrm{c}}$ Deutsches Elektronen-Synchrotron, D-22607 Hamburg, Germany ${ }^{\mathrm{d}}$ Department of Chemistry, M.V. Lomonosov Moscow State University, 119991, Moscow, Russia

* Corresponding author

Tel.: +7-925-2042939

Fax: +7-495-9390998

E-mail address: goncharoAS@gmail.com

\begin{abstract}
Iron nanowires with the diameter of ca. $40 \mathrm{~nm}$ and a length up to few dozens of microns are fabricated via templated electrodeposition using anodic aluminum oxide film as porous matrix. Despite polycrystalline structure of wires the technique allows fabrication of dense deposits with micrometer-sized single crystalline grains within AAO templates and high chemical stability towards oxidation. Nanowire arrays exhibit strong magnetization anisotropy with saturation magnetization of $180 \mathrm{emu} / \mathrm{g}$ and coercive field of $815 \mathrm{Oe}$ in direction parallel to the long axis of nanowires and 230 Oe in perpendicular direction. The effective hyperfine fields on iron atoms as extracted from Mossbauer and Nuclear Forward Scattering of sample in demagnetized state indicates slight deviation of magnetization vector $\left(\sim 6^{\circ}\right)$ from nanowire long
\end{abstract}


axis appearing probably due to curling of magnetic moments by antisymmetric exchange interactions at the surface of nanowires.

Keywords: iron; templated electrodeposition; magnetic nanowires; curling; Mossbauer spectroscopy; Nuclear Forward Scattering, antisymmetric exchange.

\section{Introduction}

Magnetic nanowires are commonly considered as a unique system for a number of practical applications including magnetic data recording media, magnetic markers, labelling and separation of the materials, rheology sensors, micro- and nanofluidics, etc $[1,2]$. Despite a number of models have been developed for the theoretical description of magnetic nanostructures in external magnetic fields [3, 4], experimental data on the arrangement of magnetic moments in such systems and its relation to volumetric magnetic characteristics of particles are still scarce. Additional ambiguity is introduced by the breaking of crystal symmetry on the surface of nanostructures inducing antisymmetric exchange interactions. The elucidation of the magnetic spins arrangement in nanostructures necessitates the complimentary analysis of data obtained by volumetric magnetic measurements with that reflecting orientation of magnetic moments such as neutron scattering, Ferromagnetic resonance (FMR) or Mossbauer spectroscopy. This task is especially challenging as it requires preparation of stable magnetic nanostructure arrays free of magnetic impurities [5].

Among common magnetic materials iron nanostructures are representative model systems exhibiting high magnetic moment, large magnetic permeability and low critical diameter [4-6]. Earlier magnetic structure of iron nanowire arrays has been examined by magnetic susceptibility measurements and Mossbauer spectroscopy in external magnetic fields applied along and perpendicular to nanowire axis [3, 7-9]. It has been found that nanowire shape anisotropy dominates the overall magnetic anisotropy in Fe, resulting in alignment of magnetization along nanowire long axis [3, 7-10]. Mossbauer spectra have illustrated well pronounced angle 
dependence of second and fifth resonances, and have enabled to deduce longitudinal and transverse demagnetizing field components from the overall effective field on iron as a function of the applied field. Unfortunately quantitative treatment of Mossbauer data has been impeded by the presence of para- or superparamagnetic iron components in the sample down to the temperatures of $10 \mathrm{~K}$, and insufficient quality of spectral data [8].

Therefore here we have focused on the fabrication of Fe/AAO nanocomposites by templated electrodeposition and the determination of crystal structure and magnetic properties of iron nanowire arrays.

\section{Experimental part}

\subsection{Preparation of porous alumina templates}

Porous anodic aluminum oxide (AAO) films were prepared by anodization of high-purity aluminum foils $(99.99 \%, 0.1 \mathrm{~mm}$ thick) in $0.3 \mathrm{M}$ oxalic acid at constant voltage of $40 \mathrm{~V}$. Prior to anodization aluminum was electropolished with an aqueous solution of $\mathrm{H}_{3} \mathrm{PO}_{4}(880 \mathrm{ml} / \mathrm{l})$ and $\mathrm{CrO}_{3}(185 \mathrm{~g} / \mathrm{l})$ at $80{ }^{\circ} \mathrm{C}$ in an impulse mode. 40 impulses for $3 \mathrm{~s}$ were imposed at an anodic current density of $0.45 \mathrm{~A} \cdot \mathrm{cm}^{2}$. In order to obtain templates with narrow pore size distribution, two-step anodization was utilized as described elsewhere [11, 12]. The anodization was carried out in two-electrode cell at $0-5{ }^{\circ} \mathrm{C}$ using $\mathrm{Pt}$ wire as a counter electrode. During anodization the electrolyte was constantly stirred. After the first anodization for $12 \mathrm{~h}$ the alumina film was selectively etched away in an aqueous solution of $\mathrm{H}_{3} \mathrm{PO}_{4}(35 \mathrm{ml} / \mathrm{l})$ and $\mathrm{CrO}_{3}(20 \mathrm{~g} / \mathrm{l})$ at $70{ }^{\circ} \mathrm{C}$ for 25 min. After the second anodization under the same conditions for $24 \mathrm{~h}$ AAO with ordered porous structure and uniform pore size distribution was obtained. The unreacted aluminum was removed in $0.5 \mathrm{M} \mathrm{CuCl}_{2}$ aqueous solution in $5 \mathrm{wt} \% \mathrm{HCl}$, and then the pore bottoms were opened by chemical etching in $25 \mathrm{wt} . \% \mathrm{H}_{3} \mathrm{PO}_{4}$ aqueous solution with electrochemical detection of the moment of pore opening [M. Lillo, D. Losic, Pore opening detection for controlled dissolution of barrier oxide layer and fabrication of nanoporous alumina with through-hole 
morphology// Journal of Membrane Science, 2009, V. 327, P. 11-17, 12]. To provide electrical contact, a layer of $\mathrm{Au}$ (ca. $100 \mathrm{~nm}$ thick) was deposited after barrier layer removal onto the bottom side of AAO by magnetron sputtering using Q150T ES (Quorum Technologies) coater.

\subsection{Iron electrocrystallization}

Iron was electrodeposited from solution containing $\mathrm{FeSO}_{4}(0.5 \mathrm{M}), \mathrm{Na}_{2} \mathrm{SO}_{4}(0.5 \mathrm{M})$, $\mathrm{H}_{3} \mathrm{BO}_{3}(0.4 \mathrm{M})$ and $\mathrm{C}_{6} \mathrm{H}_{8} \mathrm{O}_{6}(0.006 \mathrm{M})$. Electrodeposition was carried out in three-electrode cell with a bottom working electrode at room temperature using PGSTAT N302 (Autolab) potentiostat. The counter electrode was a $\mathrm{Pt}$ wire ring, and the reference electrode was a saturated $(\mathrm{KCl}) \mathrm{Ag} / \mathrm{AgCl}$ electrode connected to the cell via Luggin capillary.

\subsection{Samples characterization}

The characterization of morphology and elemental analysis of the samples was performed using scanning electron microscope (SEM) Supra 50 VP (LEO) equipped with $80 \mathrm{~mm}^{2} \mathrm{X}-\mathrm{Max}$ SDD (Oxford Instruments) microanalytical system.

High resolution transmission electron microscopy (HRTEM) images, selected area electron diffraction (SAED) patterns and energy-dispersive X-ray spectroscopy (EDS) of single iron nanowires were recorded using Libra 200 MC (Carl Zeiss) transmission electron microscope operated at $200 \mathrm{kV}$ acceleration voltage.

The quantitative determination of Fe content in electrodeposits was carried out by massspectrometry of solutions obtained by dissolution of deposits in nitric acid. Elan DRC II (Perkin Elmer) mass-spectrometer with the ionization of probe by inductively coupled Ar plasma was used.

The magnetic properties of nanocomposite Fe/AAO were studied by a vibrating sample magnetometer (VSM, Lakeshore) at room temperature.

The Mossbauer spectra were measured on an MS1104Em spectrometer operating in the constant acceleration mode with a triangular form of the Doppler velocity. The source was ${ }^{57} \mathrm{Co}$ nuclei in the Rh matrix. The Mossbauer spectrometer was calibrated at room temperature using 
an $\alpha-\mathrm{Fe}$ standard absorber. The spectra were fitted by a least squares minimization algorithm and Lorentzian line shapes to obtain the values of hyperfine field $\left(H_{\text {eff }}\right)$, isomer shift $(\delta)$, quadrupole splitting $(Q)$, line width $(G)$ and relative area $(A)$.

Nuclear Forward Scattering (NFS) experiments were carried out at P01 beamline of the PETRAIII light source (DESY, Germany). The measurements were performed at room temperature with X-ray beam aligned along the nanowires with and without external magnetic field of 4000 Oe applied along the X-ray beam. The spectra were fitted by the conventional routine [13].

\section{Results and Discussion}

\subsection{Templated electrodeposition}

According to a number of electrochemical studies $[14,15]$ an efficiency of iron electrodeposition process and the ability to form dense iron deposits with large crystal sizes depends strongly on $\mathrm{Fe}^{2+} / \mathrm{Fe}^{3+}$ equilibrium in electrolyte solution. Unfortunately most of electrolyte compositions suggested in the literature do not possess adequate stability towards oxidation. In order to minimize the concentration of $\mathrm{Fe}^{3+}$ in the electrolyte solution and to increase in electrolyte stability towards oxidation by air, ascorbic acid $(0.006 \mathrm{M})$ was added.

Cyclic voltammograms (CVA) obtained for chosen electrolyte is given in Fig. 1. For bare Au substrate low cathodic current starts at $-0.67 \mathrm{~V}$. At more negative potentials current density grows exponentially and comes to saturation at $\sim-0.95 \mathrm{~V}$. Higher overpotentials lead to increasing hydrogen evolution, which obviously would negatively affect the quality of deposits. In case of AAO-based porous electrode similar behavior of CVA curve was observed. The diminishes of deposition current as compared to bare gold substrate corresponds to the screening of the part of electrode surface by the walls of porous matrix and/or diffusion limitations of electroactive species within nanopores. The metal growth rate evaluated from current transient at deposition potential of $-0.95 \mathrm{~V}\left(2.1 \cdot 10^{-8} \mathrm{~mol} \cdot \mathrm{cm}^{-2} \cdot \mathrm{s}^{-1}\right)$ stays rather close to $\mathrm{Fe}_{a q}^{2+}$ diffusion flux of $7.6 \cdot 10^{-8} \mathrm{~mol} \cdot \mathrm{cm}^{-2} \cdot \mathrm{s}^{-1}$ as directly measured by ICP mass-spectrometry. Notably that the 
resulting diffusion coefficient of $\mathrm{Fe}_{a q}^{2+}$ in AAO channels $\left(1.5 \cdot 10^{-6} \mathrm{~cm}^{2} \cdot \mathrm{s}^{-1}\right)$ is far less than volume diffusion coefficient of iron in water solutions $\left(6 \cdot 10^{-6} \mathrm{~cm}^{2} \cdot \mathrm{s}^{-1}\right)[16]$, which was earlier attributed to suppression of diffusion by electric double layer in nanochannels [17]. Therefore the shift of deposition potential from $-0.95 \mathrm{~V}$ to more negative values does not result in the growth of the deposition current of iron due to the diffusion-limited regime, but leads to the substantial increase in hydrogen evolution. Thus a deposition potential of $-0.95 \mathrm{~V}$ was chosen for the fabrication of iron nanowires in AAO template. According to ICP MS measurements the value of current efficiency during deposition into anodic alumina template was determined as $80.7 \pm 1.7 \%$

Fig. 2 illustrates SEM images of the cross-section of Fe/AAO nanocomposite. The bright region of nanocomposite in Fig. 2a corresponds to the part of porous template filled by iron. It is clearly seen that the use of chosen electrolyte and deposition conditions results in well-defined flat growth front, which ensures a uniform filling of the pores. The length of fabricated wires is ca. $36 \mu \mathrm{m}$, resulting in high aspect ratio of nanostructures of almost 1000. The average filling factor calculated from charge spent for electrodeposition and the total volume of pores (pore diameter: $40 \mathrm{~nm}$; interpore distance: $100 \mathrm{~nm}$; porosity 15\% [18]) is equal to 52\%, whereas in the assumption that iron is almost absent in upper part of the nanocomposite (Fig. 2a) the filling factor approaches 79\%. High magnification SEM image (Fig. 2b) displays individual nanowires in AAO matrix. Rather small amount of nanowires visible in the microphotograph is due to the breaking of the nanocomposite in two parts during the preparation of cross-section. We should stress that in this regime of SEM only protruding ends of nanowires are clearly seen, whereas metal in the pores is hardly detected.

\subsection{Morphology and crystal structure of Fe nanowires}

According to the transmission electron microscopy data for individual nanowires after the dissolution of matrix in $3 \mathrm{M} \mathrm{NaOH}$, the nanostructures possess polycrystalline nature (Fig. 3). 
Well-defined wire cores are covered with an amorphous shell (Fig. 3a), which, however can result from the oxidation of wires during sample preparation procedure. Selected-area electron diffraction pattern acquired with $500 \mathrm{~nm}$ diaphragms illustrates prevalence of $\alpha$-Fe phase with micrometer-size grain sizes (Fig. 3a). Secondary low-intensity reflections can be ascribed to nanosized iron oxides. EDS mapping of individual wire indicates oxygen contamination on wire surface, with Fe:O ratio reaching 70:30 (Fig. 3b,c). We, however address the presence of oxide shell to microscopy sample preparation, involving unprotected wires exposure to strongly alkaline solution and then air for several hours. This conclusion is supported by VSM and Mossbauer spectroscopy measurements of the Fe/AAO nanocomposite where no substantial amount of oxygen-containing phases was found.

\subsection{Magnetic properties of Fe/AAO nanocomposite}

According to VSM measurements Fe/AAO nanocomposite indicates a large anisotropy of magnetic properties (Fig. 4), characteristic for magnetic metal nanowires synthesized in AAO matrix [19]. Coercive field in longitudinal direction attains 815 Oe, whereas in case of perpendicular magnetization it falls down to 230 Oe, indicating a serious role of demagnetizing field and shape anisotropy on the magnetic properties of nanocomposite. Saturation magnetization per iron atom, as calculated from the total iron content in the sample, attains 180 emu/g, which is comparable to the saturation magnetization of the bulk $\alpha-\mathrm{Fe}$.

Mossbauer spectrum of Fe/AAO nanocomposite corresponds well to $\alpha$-Fe phase, with component parameters $H_{\text {eff }}=330 \mathrm{kOe}, \delta=0 \mathrm{~mm} \cdot \mathrm{s}^{-1}, Q=0 \mathrm{~mm} \cdot \mathrm{s}^{-1}, G=0.27 \mathrm{~mm} \cdot \mathrm{s}^{-1}$ (Fig. 5). No well-defined signs of the substantial amount of oxygen-containing phases have been found in the spectrum even after six months exposure to air. This suggests formation of dense iron deposit under chosen conditions protecting iron core in AAO matrix from oxidation by air. For the zerofield spectrum, the second and fifth absorption peaks in the sextet almost disappear. In 
magnetically split spectra, the relative intensities of the $2^{\text {nd }}$ and $5^{\text {th }}$ lines (corresponding to the $\Delta m=0$ nuclear transitions) are given by $I_{2,5}=4 \sin ^{2} \theta /\left(1+\cos ^{2} \theta\right)$, where $\theta$ is the angle between the magnetic moment and beam direction [20]. Experimental intensity ratio for 1-3 transitions equals to $3: 0.05: 1$, corresponding to $6.4^{\circ}$ average field tilt to the long axis of nanowires in the demagnetized state.

Detailed analysis of Mossbauer spectrum enables to extract paramagnetic (at room temperature) iron (II) contribution with $\delta=0.63 \mathrm{~mm} \cdot \mathrm{s}^{-1}, Q=1.28 \mathrm{~mm} \cdot \mathrm{s}^{-1}, G=0.75 \mathrm{~mm} \cdot \mathrm{s}^{-1}$ and $A<3 \%$, indicating possible surface oxidation of iron. Slight asymmetry of $1^{\text {st }}$ and $6^{\text {th }}$ lines in $\alpha-F e$ sextet could also be addressed to hyperfine field distribution appearing due to surface magnetic oxides or boundary effects.

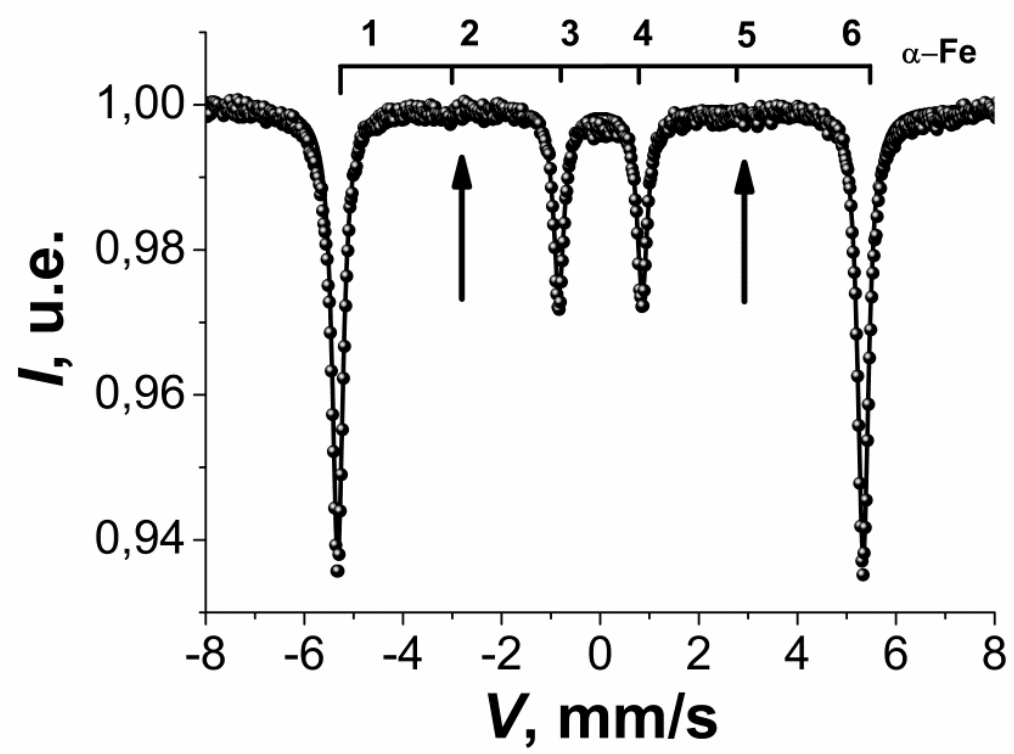

Fig. 5 Mossbauer spectrum of Fe nanowires embedded in the AAO template recorded after the exposure of nanocomposite in air for six moths

The results of Mossbauer spectroscopy and of the magnetometry measurements were confirmed by the characterization of the sample by the Nuclear Forward Scattering (NFS) of synchrotron radiation. This method is the time analogue of the Mossbauer spectroscopy. Due to the linear polarization of the synchrotron radiation beam, not only axis of the spin orientation but 
also direction of the total spin can be extracted [21]. Fig. 6 shows NFS spectra recorded at P01 beamline of the PETRAIII light source without (A) and with (B) external magnetic field of 4000 Oe. The experimental data were fit by the two components of the $\alpha$-Fe phase in the antiparallel direction of the spins with angle showing deviation of the spins from long axis of the nanowires (X-ray beam direction) as a free parameter. Other free parameters were the ratio of the components, hyperfine magnetic field and effective thickness. The deviation angle can be considered as a real angle of the spin deviation or as an effective parameter describing misalignment of the spins. In particular, completely isotropic spins alignment is described by the deviation angle of $54.7^{\circ}$ and ratio of two components $1: 1$. The fit of the data were performed by MOTIF software [22]. Without magnetic field the amplitude of the hyperfine magnetic field of $337 \mathrm{kOe}$ was obtained. The deviation angle was $6 \pm 2^{\circ}$ which is in accordance to the results of Mossbauer spectroscopy and the ratio of contributions in positive and negative directions was 0.68:0.32. Application of 4000 Oe field leads to the complete switch of the spins to one direction as seen from the change of the time spectra between Fig. 6a and 6b. The deviation angle extracted as one of fit parameters in this case is about $8 \pm 3^{\circ}$.

Notably that the resulting $M_{\mathrm{r}}: M_{\mathrm{s}}$ ratio of 0.36 extracted from NFS data exceeds volumetric $M_{\mathrm{r}}=0.29 M_{\mathrm{s}}$ as obtained from VSM magnetometry. This indicates misalignment of a part of magnetic moments on iron atoms from nanowire axis, which stays in line with Mossbauer spectroscopy results. We ascribe this effect to slight curling of magnetic moments at the surface of nanowires.

Spin canting in nanowires can arise either from demagnetizing fields (produced by iron wire itself or by surrounding wires [23]) or antisymmetric exchange interactions appearing at the surface of nanowires. Persistence of canting in high external magnetic fields exceeding a saturation (as observed by NFS), gives a sign for strong magnetic exchange interactions to be dominating. In turn, antisymmetric exchange can be defined either by Dzyaloshinskii-Moriya interactions due to symmetry breaking at the surface of nanowires or by a biquadratic exchange 
anisotropy due to coupling of iron nanowire spins to thin oxide covering layer (invisible by Mossbauer spectroscopy). The dominating effect cannot indisputably be elucidated so far, and would stay for scope of further studies.

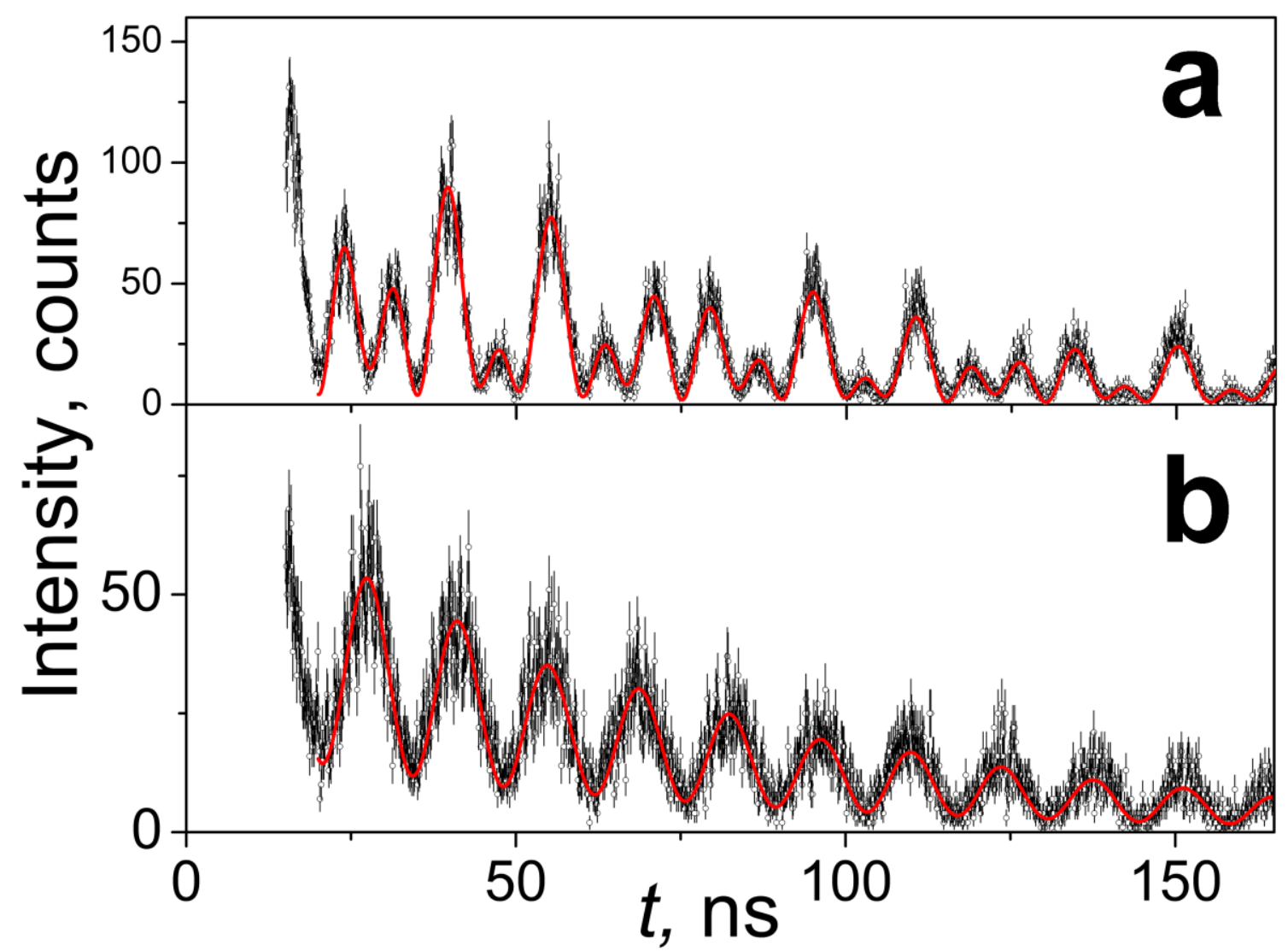

Fig. 6 NFS time spectra for Fe/AAO nanocomposite measured with synchrotron radiation beam along the nanowires without (a) and with 4000 Oe external field (b). The line shows fit of the experimental data

\section{Conclusions}

The proposed technique of iron templated electrodeposition in the pores of $55 \mu \mathrm{m}$ thick anodic aluminum oxide in the presence of stabilizing ascorbic acid results in successful fabrication of iron nanowires with diameter of $\sim 40 \mathrm{~nm}$ and aspect ratio of ca. 1000. Despite polycrystalline structure of wires, dense iron deposits with micrometer-sized single crystalline grains possess high chemical stability towards oxidation within AAO templates. Nanowire arrays exhibit strong magnetization anisotropy with saturation magnetization of $180 \mathrm{emu} / \mathrm{g}$ and coercive field of 815 Oe in direction parallel to the long axis of nanowire and 230 Oe in perpendicular 
direction as defined by demagnetization field. Mossbauer and Nuclear Forward Scattering measurements of Fe/AAO nanocomposite in demagnetized state indicates $\sim 6^{\circ}$ deviation of magnetization vectors from the long axis of nanowires appearing probably due to curling of magnetic moments by antisymmetric exchange arising either from Dzyaloshinskii-Moriya interactions at the surface of nanowires or biquadratic exchange coupling to a surface oxide layer.

\section{Acknowledgments}

This work is supported by the Russian Science Foundation (Grant No. 14-13-00809). Part of experiments was carried out using the scientific equipment purchased by M.V. Lomonosov Moscow State University Program of Development. The PETRA III light source is acknowledged for provision of synchrotron radiation beam time at the beamline P01.

\section{References}

1. Rao CNR, Deepak FL, Gundiah G, Govindaraj A (2003) Inorganic nanowires. Progress in Solid State Chemistry 31 (1): 5-147

2. Ramazani A, Kashi M A, Isfahani V B, Ghaffari M (2010) The influence of crystallinity enhancement on the magnetic properties of ac electrodeposited Fe nanowires. $\mathbf{J}$ Appl Phys A 98:691-697

3. Paulus PM, Luis F, Kroll M, Schmid G, de Jongh LJ (2001) Low-temperature study of the magnetization reversal and magnetic anisotropy of $\mathrm{Fe}, \mathrm{Ni}$, and $\mathrm{Co}$ nanowires. J Magn Magn Mater 224:180-196 
4. Sun L, Hao Y, Chien C-L, Searson PC (2005) Tuning the properties of magnetic nanowires. IBM J- Res: \& Dev- 49:-79-102:

5. Cui C, Yang W, Sun J, Zhang Q (2011) Electrodepositing fabrication and microstructures of the Fe nanowires with a preferred orientation. Superlatt Microstruct $50: 628-633$

6. Eliseev AA, Vyacheslavov AS, Lukashin AV, Tretyakov YD, Suzdalev IP, Maximov YV, Goernert P (2006) Iron-containing nanocomposites based on ZSM-5 zeolite. International Journal of Nanoscience 5 (04n05):-459-463

7. Cornejo DR, Padron-Hernandez E (2007) Study of magnetization process in ordered Fe nanowire arrays. J Magn Magn Mater 316:e48-e51

8. Zhan Q, He W, Ma X, Liang Y, Kou Z, Di N, Cheng Z (2004) Applied field Mössbauer study of shape anisotropy in Fe nanowire arrays. Appl Phys Lett 85, 4690-4692

9. Peng Y, Zhang HL, Pan SL, Li HL (2000) Magnetic properties and magnetization reversal of $\alpha$-Fe nanowires deposited in alumina film. J Appl Phys 87:7405-7408

10. Zhan Q, Chen Z, Xue D, Li F, Kunkel H, Zhou X, Roshko R, Williams G (2002) Structure and magnetic properties of Fe-Co nanowires in self-assembled arrays. Phys Rev B 66:134436-1-134436-6

11. Napolskii KS, Roslyakov IV, Romanchuk AY, Kapitanova OO, Mankevich AS, Lebedev VA, Eliseev AA (2012) Origin of long-range orientational pore ordering in anodic films on aluminium. J Mater Chem 22:11922-11926

12. Petukhov DI, Napolskii KS, Berekchiyan MV, Lebedev AG, Eliseev AA (2013) Comparative study of structure and permeability of porous oxide films on aluminum obtained by single-and two-step anodization. ACS Applied Materials \& Interfaces 5: 7819-7824 
13. Herlitschke M, Disch S, Sergueev I, Schlage K, Wetterskog E, Bergstrom L, Hermann R P (2016) Spin disorder in maghemite nanoparticles investigated using polarized neutrons and nuclear resonant scattering. J Physics: Conference Series 711:012002

14. Grujicic D, Pesic B (2005) Iron nucleation mechanisms on vitreous carbon during electrodeposition from sulfate and chloride solutions. Electrochim Acta 50:4405-4418

15. Masanobu Izaki. Electrodeposition of iron and iron alloys. Modern Electroplating, Fifth Edition (Edited by Mordechay Schlesinger and Milan Paunovic, 2010, John Wiley \& Sons, Inc.

16. Lide DR (2003) CRC Handbook of Chemistry and Physics, Taylor \& Francis

17. Napolskii KS, Roslyakov IV, Eliseev AA, Petukhov DI, Lukashin AV, Chen S-F, Liu CP, Tsirlina GA (2011) Tuning the microstructure and functional properties of metal nanowire arrays via deposition potential. Electrochim Acta 56:2378-2384

18. Petukhov DI, Berekchiian MV, Pyatkov ES, Solntsev KA, Eliseev AA (2016) Experimental and Theoretical Study of Enhanced Vapour Transport Through Nanochannels of Anodic Alumina Membranes in Capillary Condensation Regime. J Phys Chem C, 120 (20): 10982-10990

19. Napolskii KS, Eliseev AA, Yesin NV, Lukashin AV, Tretyakov YD (2007) Ordered arrays of $\mathrm{Ni}$ magnetic nanowires: Synthesis and investigation. Physica E: Lowdimensional Systems and Nanostructures 37:178-183

20. Preston RS, Hanna SS, Heberle J (1963) Mossbauer effect in Metallic Iron. Phys- Rev$130: 2207-2218$

21. Rohlsberger R (2005) Nuclear Condensed Matter Physics with Synchrotron Radiation. Basic Principles, Methodology and Applications. Book. Springer Tracts in Modern Physics. V. 208

22. Shvyd'ko YV, (2000) MOTIF: Evaluation of time spectra for nuclear forward scattering, Hyperfine Interactions, 125 (1): 173-188 
23. Grigoriev SV, Grigoryeva NA, Napol'skii KS, Chumakov AP, Eliseev AA, Roslyakov IV, Eckerlebe H, Syromyatnikov AV (2011) Arrays of interacting ferromagnetic nanofilaments: Small-angle neutron diffraction study. Jetp Lett 94 (8):635-641. 